\title{
Review of Face Recognition Techniques
}

\author{
Mandeep Kaur \\ Research Scholar, \\ Dept. of Computer Science \\ Global Institutes \\ Amritsar, India
}

\author{
Jasjit Kaur \\ AP, Dept. of Computer Science \\ Global Institutes \\ Amritsar, India
}

\begin{abstract}
Face Recognition is used in order to ensure authentication in terms of feature verification. Techniques are defined to identify faces under different situations. This paper conducts a survey of techniques which are available for face detection. Recognition is possible in case features are extracted from the presented face images. For this purpose feature extraction mechanisms like discrete wavelet transformation (DWT), SIFT, linear discriminate analysis (LDA), principal component analysis (PCA) are commonly used. Analysis process indicates that hybrid approach with discrete wavelet transformation produces better results. Comparative study of literature is also presented through this work.
\end{abstract}

\section{Keywords}

Face Recognition, Feature Extraction, DWT, SIFT, LDA, PCA.

\section{INTRODUCTION}

Face recognition is computer application capable of identifying or verifying a person from the image or video source. Legions of facial expressions exist and hence identification process is complex in nature. Automated approach is suggested through many researchers to detect facial recognition. Automated approach uses image processing techniques to detect and drive conclusion regarding face detection. Quality of image is critical in face recognition. The quality can be enhanced by the use of image processing techniques. Feature of interest can be extracted and improved by the use of image processing techniques[1]. Image distortion is generally caused through presence of noise within the image. Noise handling mechanisms hence is vital. Common noise present within the image is Gaussian noise. To handle such noise conditional likelihood technique is most useful[2]. Contrast enhancement techniques is used in image processing to increase clarity within the image which are distorted due to camera movement, darkness etc. The histogram equivalence techniques are useful in such situations.

\subsection{Face detection techniques based on different model}

Techniques describing face extraction process is described as follows:

- Knowledge based face detection: [3] In this technique the face can be detected based on various rules that translate knowledge about the face. This approach examines the face in hierarchal levels. The first level defines the candidates that are based on the intensity of the image, second level extract the edges in the image and the last level the image can be grouped in face and non-face image.
- Feature invariant technique: [4],[5]In this technique edge detection and morphological operations are to be used for detection of local features in the face. The various approaches are to be utilised for describing relational ships between features such as neural network graph matching and statistical model.

- $\quad$ Structure based face detection: [6]In this pixels are to be analysed according to skin or nonskin area. The various approaches are to be utilised for grouping and connected component analysis. Also it uses the statistical models of the shape of face appeared on the image.

- Templates based technique: [7]In this technique for describing the facial features several standard patterns are to be utilised. These patterns are to be analysed the edges and silhouettes of the images for recognise the face.

- Appearance based technique: [8]In this technique from large set of images the faces are to be detected using statistical and machine learning approaches.

\subsection{Techniques for feature extraction}

1. Discrete wavelet transform

[9]DWT is widely used in numerical and functional analysis. In these areas wavelets are considered to be discretely distributed. DWT has advantage that both location and frequency information is considered. DWT has advantage over Fourier transformation since it has temporal resolution. The concept of wavelet is simple. They are used for multistage analysis process. Description of multistage wavelet is described considering the example as

Example 1

The sequence of wavelets are considered using $n=2^{3}$

$\mathrm{y}=\{1,1,2,3,1,3,2,2\}$

Consider vectors $\mathrm{P}$ and $\mathrm{L}$ computed through algorithm for multistage which can be applied as follows

$$
\begin{aligned}
& \text { 1. } P_{I_{-1, J}}=\frac{1}{\sqrt{2}}\left(L_{I, 2 K}-L_{I, 2 K_{-1}}\right) \\
& \text { 2. } L_{I-1, J}=\frac{1}{\sqrt{2}}\left(L_{I, 2 K}+L_{I, 2 K_{-1}}\right) \\
& \text { 3. } I=I-1 \\
& \text { 4. } I f \text { i=0 then stop else move to step } 1
\end{aligned}
$$

The basic ideas behind wavelets are portrayed through the above listed algorithm. The algorithm provides basic understanding of the wavelets or provides compact structure analysis of stored information. 
Discrete wavelet transformation is also described in terms of energy. The energy conservation can be described considering the following example.

Example 2

Sequence of pattern can be considered in which energy is stored. The pattern can be analyzed using following

$$
\begin{gathered}
\|y\|^{2}=\left\{1^{2}+1^{2}+2^{2}+3^{2}+1^{2}+3^{2}+2^{2}+2^{2}\right\} \\
\|y\|^{2}=29
\end{gathered}
$$

Transform coefficient can be calculated using following equation

$$
\begin{gathered}
|| Y \& T_{0,0}||^{2}=0^{2}+\left(\frac{\sqrt{2}}{2}\right)^{2}+(\sqrt{2})^{2}+(-\sqrt{2})^{2}+\left(\frac{3}{2}\right)^{2} \\
+(-1)^{2}+\left(-\frac{\sqrt{2}}{4}\right)^{2}+\left(\frac{13 \sqrt{2}}{4}\right)^{2} \\
\left.|| Y \& T_{0,0}\right|^{2}=29
\end{gathered}
$$

Since both values are equal hence energy is conserved.

\section{Correlation}

[10]Face detection is complex since many distinct expressions are involved. The coordinate analysis is conducted to determine match or mismatch expression. For this purpose correlation is useful. Correlation expresses relationship between pixels through position values including $\mathrm{x}$ and $\mathrm{y}$. Correlation is expressed with the help of correlation coefficient indicated with $\mathrm{r}$. Value of $\mathrm{r}$ lies between 0 and 1 . Correlation coefficient is best described considering the following example.

$\begin{array}{cc}\mathrm{X} & \mathrm{Y} \\ \mathrm{X} 1 & \mathrm{Y} 1 \\ \mathrm{X} 2 & \mathrm{Y} 2 \\ \mathrm{X} 3 & \mathrm{Y} 3 \\ \mathrm{X} 4 & \mathrm{Y} 4 \\ \mathrm{X} 5 & \mathrm{Y} 5 \\ --- & -- \\ --- & -- \\ \mathrm{Xn} & \mathrm{Yn}\end{array}$

Then correlation coefficient is calculated using following equation.

$\mathrm{r}_{\mathrm{xy}}=\sum \frac{\left(x_{i}-x^{-}\right)\left(y_{i}-y^{-}\right)}{\sqrt{\sum\left(x_{i}-x^{-}\right)^{2} \sum\left(y_{i}-y^{-}\right)^{2}}}$

\section{Principal component analysis}

[11]Correlation mechanism is complex and high cost is encountered as complex images are handled. In order to resolve the problem PCA method is used. PCA is a statistical procedure that uses orthogonal transformation to convert possibly correlated values into set of non correlated linear values known as principal components. It utilizes the set of eigenvalues that are builds from the set of training data sets. From these eigenvalues the training face images have been calculated which are arranged for finding the most variance in image. After this the Euclidean distance from the input face has been calculated for each eigenvalues. This can classified the image into parts based on Euclidean distance. The weighted sum of eigenfaces represented by text face images projected on to the space expanded by eigenfaces. The faces can be identified by these weights.

The following is way through which the correlated values has been calculated:

$$
\begin{gathered}
\mu=\frac{1}{m} \sum_{n=1}^{m} x_{n} \\
C=\frac{1}{m} \sum_{n=1}^{m}\left(x_{n}-\mu\right)\left(x_{n}-\mu\right)^{T}
\end{gathered}
$$

4. Linear discriminant analysis

[3]Linear Discriminant analysis is useful to determine combined features that do the separation of the classes. The length and complexity associated with the calculations are reduced using LDA approach. The dimensionality reduction and classification of face recognition is accomplished using least time and space complexity. Distortion within the image is common. This is also accomplished through LDA.

Mathematically, a set of $\mathrm{n}$ dimensional vectors $\mathrm{x}_{\mathrm{i}}, \mathrm{x}_{2},-------, \mathrm{x}_{\mathrm{n}}$ belongs to 1 classes of faces.

$\operatorname{Max} \frac{w^{7} S_{n} w}{w^{7} S_{w} w}$

Where

$\mathrm{S}_{\mathrm{n}}=\sum_{i-1}^{n} n_{i}\left(u^{i}-u_{\text {total }}\right)\left(u^{i}-u_{\text {total }}\right)$

$\mathrm{S}_{\mathrm{w}}=\sum_{i=1}^{n} \sum_{j=1}^{n}\left(x^{i}-u^{i}\right)\left(x^{i}-u^{i}\right)^{i}$

$\mathrm{U}$ is the mean of training images presented to the simulation. $S_{w}$ is within the scatter matrix and $S_{n}$ is between class scatter matrix.

5. Structural similarity index matrix

[12]In this the reference image has to be compared with the text image for finding its quality. The similarity between them can be measured using three comparisons component: luminance contrast and structure. The structural similarity index matrix can be measured using following formula:

$$
s(x, y)=f(l(x, y), s(x, y) . c(x, y))
$$

The components are independent of each other. This wavelet transform provides the simultaneous localization in time and frequency domain. The technique provides capability of revealing various aspects like trends, breakdown points, and discontinuities in higher derivatives and self similarity for analyzing the data. The PCA deal with low noise sensitivity and the LDA has deal with high dimensional data. So in this method we overcome the disadvantage of the PCA and LDA methods and provide the scalar methods for analyzing the image. The $2 \mathrm{~d}$ image condenses into single scalar value which is insensitive to the variations in brightness and contrast across the image.

The detailed study of above methods is conducted. The techniques are then merged in order to find optimal 
mechanism for face recognition. The proposed scheme combines DWT and LDA method in which result is obtained through decomposition of metrics in four details sub bands. The information obtained is approximation details. The reduced image information is presented to PCA to obtain principal components and reduces dimensionality for storing. The proposed approach is capable of reducing image registration and is highly sensitive to skewing, pin cushioning and vignette that inevitably occurs in images.

Next section describes literature survey describing worth of the study.

\section{LITERATURE SURVEY}

The literature survey is conducted to suggest validity of proposed work. Also current level of research is also reflected through this work.

(Gaidhane, Hote, and Singh 2014) [8]researched approach uses simple algorithm in order to detect faces out of many faces. It utilizes polynomial coefficient, covariance matrix and algorithm based on Eigen values. The symmetry matrix is calculated based on common features extracted from the image dataset. The nullity is calculated to determine dissimilar features extracted. The nullity for dissimilar image is small. Experimental results show validation of this approach.

(Mehta, Yuan, and Egiazarian 2014) [13]proposes face detection based on extraction of directional and texture feature from the image. The directionality is associated with image is extracted using local polynomial approximation technique (LPA) which is directional filter at multiple scales. Singular valued decomposition approach is used to reduce dimensionality. The proposed approach also compares this technique against other state of the art technique to prove validly of research.

(Seo and Park 2014) [14]handles 2D images be extracting features of the image. Features extraction mechanism uses SIFT (Scale invariant Feature transform). SIFT is used to represent local features as a descriptor. Variation in facial images is identified by the use of probability density function calculated from SIFT. Benchmark datasets are used for detection of facial variations through suggested technique.

(Wan and Aggarwal 2014) [15] used to address the problem of identifying spontaneous facial expression. The proposed method utilizes specificity and sensitivity model that contains mislabeled expression existing in training data with different annotators. The expectation-maximization (EM) procedure is deployed to estimate the true label of expression by computing the likelihood with a metric. An adaptive online metric learning is proposed to solve optimal positive semidefinite metric with the steepest descent method. The method performs the classification by simple means of voting. The experiment results show better result and performance.

(Luan et al. 2014) [11]describes the fact that face recognition can be accomplished though variations such as illumination and occlusion. The proposed method deals with these variations. This method has advantage that feature selection procedure is not utilized hence complexity is reduced. The descriptors namely sparsity and smoothness are used in this technique. After this recognition process is used to detect the face. First descriptor computes the sparsity of image matrix to find the degradation of the image with noise and error. second descriptor utilize ingredient domain. The true identity of the image is identified with the help of these descriptors. The proposed method efficiently determines face expression.

(Dpdrnd et al., n.d.) [16]Research utilizes correlation in order to detect faces from noise. The proposed scheme of thing utilizes reverse correlation mechanism to detect falsie images. The results obtained suggest that face detection performance increases linearly with number of false image detection. Results also suggest both human and machine face detection algorithms make similar errors hence both AdaBoost and EigenFace are good approximation for human face processing.

(An et al. 2009) [3]utilizes LBP method for face detection. The face detection process uses scalable window which divides the region into subparts. Features are extracted using MapLBP approach to describe local structure of the face. This approach produces better result and can process images with 16 frames per second.

(Samadi and Pourghassem 2013) [17]proposes approach which is based on LDA approach. In this paper, a practical algorithm in children classification from adults by their facial image is proposed. In this algorithm, statistical modeling of the face is used to extract the age dependent face features and then by applying Linear Discriminant Analysis (LDA) on the face parameters, useful specifications are extracted. This approach achieved $85 \%$ accuracy in face detection.

(Dave and Tech, n.d.) [6]considers Principal Component Analysis approach to detect facial expression. Shot boundary detection is achieved through PCA approach. This technique specifically provides or detects both gradual shot transitions and the cut presents in video. Haar Wavelet Transform used to detects shot boundary. Frame Correlation threshold require to set so that shot boundaries easily can be detected.

(Peng and Member 2013) [18]proposed a method based on Principal component analysis. A dedicated rule-based algorithm is first implemented to detect four facial feature points which are used to align the input face images and partition the face region into four components. Based on each group of facial components obtained from the training dataset, an eigen subspace is constructed through principal component analysis(PCA). The dataset of 464 images is used and high degree of accuracy is achieved through this approach.

The comparative analysis indicates that use of eigen values produces optimal result in case of face detection. 
Table 1: Comparative study from literature

\begin{tabular}{|c|c|c|c|c|}
\hline Parameters & Journal/Conference & Technique & Error Rate & Recognition Rate \\
\hline $\begin{array}{l}\text { (Luan et al. } \\
\text { 2014) }\end{array}$ & ELSEVIER & PCA & 0.003 (Assumed) & $95.06 \%$ \\
\hline $\begin{array}{l}\text { (Seo and } \\
\text { Park 2014) }\end{array}$ & ELSEVIER & SIFT & Not Specified & $\begin{array}{l}96 \% \text { obtained since total is given to be } 0.969 \text {. } \\
\text { in terms of percentage } 0.969^{*} 100=96 \%\end{array}$ \\
\hline $\begin{array}{c}\text { (Mehta, } \\
\text { Yuan, and } \\
\text { Egiazarian } \\
\text { 2014) }\end{array}$ & ELSEVIER & $\begin{array}{l}\text { Scale Adaptive } \\
\text { directional and } \\
\text { textural features }\end{array}$ & $\begin{array}{c}0 \text { to } 8 \% \text { in terms of } \\
\text { noise }\end{array}$ & $95 \%$ on an average \\
\hline $\begin{array}{c}\text { (Gaidhane, } \\
\text { Hote, and } \\
\text { Singh 2014) }\end{array}$ & ELSEVIER & Eigen Values & Not specified & $99.4 \%$ \\
\hline $\begin{array}{l}\text { (Peng and } \\
\text { Member } \\
\text { 2013) }\end{array}$ & IEEE & PCA & $8.5 \%$ & $98.2 \%$ \\
\hline
\end{tabular}

\section{CONCLUSION AND FUTURE SCOPE}

Face recognition is state of the art problem present among the researchers. Human facial expressions vary with time. Automatic identification of various human facial expressions is critical in human and computer interaction. Legions of factor exist such as noise, environment, camera movement, cluttered background etc. which degrades the performance of face recognition system. The techniques of image processing involving filtering, correlation among test and training images etc. collaborated together to resolve this problem. The face recognition is the need of the hour since security and protection is greatly enhanced by the use of face detection. In order for the efficient recognition, face detection must be accurate. Distinct techniques are used to accomplish accuracy in face detection. Discrete wavelet transformation if hybridized with other techniques then recognition rate and accuracy is drastically improved.

In the future SIFT keys along with DWT can be hybridized to obtain better results.

\section{REFERENCES}

[1] "Digital Image Processing for Image Enhancement and Information Extraction."

[2] Y. I. Abramovich, O. Besson, S. Member, B. A. Johnson, and S. Member, "Conditional expected likelihood technique for compound Gaussian and Gaussian distributed noise mixtures," no. c, pp. 1-12, 2016.

[3] K. H. An, S. H. Park, Y. S. Chung, K. Y. Moon, and M. J. Chung, "Features for Face Detection Based on AdaLDA," pp. 1117-1122, 2009.

[4] T. Kaur and R. Dhir, "A Non-domination Pareto-based Scale-Invariant Approach for Face Recognition," Eur. J. Eng. Res. Sci., vol. 1, no. 2, pp. 6-13, 2016.
[5] L. Juan and O. Gwun, "A comparison of sift, pca-sift and surf," Int. J. Image Process., vol. 3, no. 4, pp. 143-152, 2009.

[6] P. Dave and M. Tech, "Study and Analysis of Face Recognition system using Principal Component Analysis ( PCA )."

[7] T. Xu, Y. Wang, and Z. Zhang, "Pixel-wise skin colour detection based on flexible neural tree," Image Process. IET, vol. 7, no. April, pp. 751-761, 2013.

[8] V. H. Gaidhane, Y. V Hote, and V. Singh, "An ef fi cient approach for face recognition based on common eigenvalues," Pattern Recognition., vol. 47, no. 5, pp. 1869-1879, 2014.

[9] C. Tippanna Madiwalar, S. K. Babu, R. K. B, and V. K. $\mathrm{R}$, "Compression Based Face Recognition Using Dwt and Svm," An Int. J., vol. 7, no. 3, pp. 444-449, 2016.

[10] Y. Xiao, Z. Cao, and T. Zhang, "Entropic thresholding based on gray-level spatial correlation histogram," in 2008 19th International Conference on Pattern Recognition, 2008, pp. 1-4.

[11] X. Luan, B. Fang, L. Liu, W. Yang, and J. Qian, "Extracting sparse error of robust PCA for face recognition in the presence of varying illumination and occlusion," Pattern Recognit., vol. 47, no. 2, pp. 495508, 2014.

[12] T. Pecchia, A. Gagliardo, C. Filaninno, P. Ioalè, and G. Vallortigara, "Metadata of the chapter that will be visualized in SpringerLink Adult-Born Neurons in the Olfactory," Behav. Lateralization Vertebr., 2012.

[13] R. Mehta, J. Yuan, and K. Egiazarian, "Face recognition using scale-adaptive directional and textural features," Pattern Recognition., vol. 47, no. 5, pp. 1846-1858, 2014. 
[14] J. Seo and H. Park, "Neurocomputing Robust recognition of face with partial variations using local features and statistical learning," Neurocomputing, vol. 129, pp. 4148,2014

[15] S. Wan and J. K. Aggarwal, "Spontaneous facial expression recognition: A robust metric learning approach," Pattern Recognition., vol. 47, no. 5, pp. 1859-1868, 2014.

[16] K. Dpdrnd, D. Q. G. Dwvxpl, X. Idfhv, H. Txdolwdwlyho, R. U. Txdqwlwdwlyho, V. V. Lv, W. R.
Dqdo, H. H. Wkdw, and W. K. H. V Vwhp, “pp-206215, 2013."

[17] A. Samadi and H. Pourghassem, "Children Detection Algorithm Based on Statistical Models and LDA in Human Face Images,” pp. 206-209, 2013.

[18] P. Peng and I. S. Member, "Efficient Face Verification in Mobile Environment Using Component-based PCA," no. Cisp, pp. 753-757, 2013. 